\title{
Observation of Phase Fluctuations in Elongated Bose-Einstein Condensates
}

\author{
S. Dettmer, D. Hellweg, P. Ryytty, J. J. Arlt, W. Ertmer, and K. Sengstock \\ Institut für Quantenoptik, Universität Hannover, Welfengarten 1, 30167 Hannover, Germany \\ D. S. Petrov ${ }^{1,2}$ and G. V. Shlyapnikov ${ }^{1,2,3}$ \\ ${ }^{1}$ FOM Institute for Atomic and Molecular Physics, Kruislaan 407, 1098 SJ Amsterdam, The Netherlands \\ ${ }^{2}$ Russian Research Center, Kurchatov Institute, Kurchatov Square, 123182 Moscow, Russia \\ ${ }^{3}$ Laboratoire Kastler Brossel, Ecole Normale Supérieure, 24 rue Lhomond, 75231 Paris Cedex 05, France
}

\author{
H. Kreutzmann, L. Santos, and M. Lewenstein \\ Institut für Theoretische Physik, Universität Hannover, Appelstraße 2, 30167 Hannover, Germany
}

(Received 28 May 2001; published 1 October 2001)

\begin{abstract}
The occurrence of phase fluctuations due to thermal excitations in Bose-Einstein condensates (BECs) is studied for a variety of temperatures and trap geometries. We observe the statistical nature of the appearance of phase fluctuations and characterize the dependence of their average value on temperature, number of particles, and the trapping potential. We find pronounced phase fluctuations for condensates in very elongated traps in a broad temperature range. The results are of great importance for the realization of BEC in quasi-1D geometries, for matter wave interferometry with BECs, as well as for coherence properties of guided atom laser beams.
\end{abstract}

DOI: $10.1103 /$ PhysRevLett.87.160406

PACS numbers: 03.75.Fi, 05.30.Jp, 32.80.Pj

Since the first experimental realization of Bose-Einstein condensation in dilute atomic gases [1], there has been enormous interest in the coherence properties of BoseEinstein condensates (BECs). In particular, the phase coherence is essential for applications of BEC in matter wave interferometry; it also sets limits on the coherence of atom lasers, and guided atom laser beams. For a trapped 3D condensate well below the BEC transition temperature $T_{c}$, recent experiments have confirmed the phase coherence; e.g., it was shown that the coherence length is equal to the condensate size $[2,3]$. However, phase coherence is not an obvious property of BEC. In particular, it is expected that low-dimensional (1D and 2D) quantum gases differ qualitatively from the 3D case in this respect [4-7]. Recently, it was shown theoretically [8] that for very elongated condensates phase fluctuations can be pronounced already in the equilibrium state of the usual 3D ensemble, where the density fluctuations are suppressed. The phase coherence length in this case can be smaller than the axial size of the sample. This is referred to as the regime of quasicondensation [9]. The detailed characterization of phase fluctuations in condensates is thus of great importance for applications of BEC, especially for recent attempts to reach BEC in elongated microcircuit geometries [12]. The temperature dependence of the coherence of an atom laser beam was studied in [13].

In this Letter we report on systematic studies of BEC of ${ }^{87} \mathrm{Rb}$ atoms in the regime of phase-fluctuating condensates. We achieved this regime in highly anisotropic traps leading to a strongly elongated shape of the condensate. We observe the phase fluctuations by measuring the density distribution of the released cloud after ballistic expansion. By varying the temperature and the aspect ratio of the trap- ping potential, we study the continuous transition from the usual 3D regime, where phase fluctuations of the condensate are low, into the regime of strong phase fluctuations. We show that a phase coherent matter wave is not a direct outcome of BEC in strongly elongated geometries but can be achieved only for very low temperatures, well below the BEC transition temperature $T_{c}$.

Fluctuations of the phase of a Bose condensate are related to thermal excitations and always appear at finite temperature. However, as shown in Ref. [8], the fluctuations depend not only on temperature but also on the trap geometry and on the particle number. Typically, fluctuations in spherical traps are strongly suppressed as the wavelengths of excitations are smaller than the size of the atomic cloud. In contrast, wavelengths of the excitations in strongly elongated traps can be larger than the radial size of the cloud. In this case, the low-energy axial excitations acquire a 1D character and can lead to more pronounced phase fluctuations, although the density fluctuations of the equilibrium state are still suppressed. Because of the pronounced phase fluctuations, the coherence properties of elongated condensates can be significantly altered as compared with previous observations. In particular, the axial coherence length can be much smaller than the size of the condensate, which can have dramatic consequences for practical applications.

The experiment was performed with Bose-Einstein condensates of up to $N_{0}=5 \times 10^{5}$ rubidium atoms in the $\left|F=2, m_{F}=+2\right\rangle$ state of a cloverleaf-type magnetic trap. Further details of our apparatus were described previously $[14,15]$. The fundamental frequencies of the magnetic trap are $\omega_{x}=2 \pi \times 14 \mathrm{~Hz}$ and $\omega_{\rho}=2 \pi \times 365 \mathrm{~Hz}$ along the axial and radial directions, respectively. Because of the highly anisotropic confining potential with an aspect 
ratio $\lambda=\omega_{\rho} / \omega_{x}$ of 26 , the condensate is already elongated along the horizontal $x$ axis. In addition, further radial compression of the ensemble by means of a superimposed blue detuned optical dipole trap is possible [16]. We performed the measurements for radial trap frequencies $\omega_{\rho}$ between $2 \pi \times 138 \mathrm{~Hz}$ and $2 \pi \times 715 \mathrm{~Hz}$ corresponding to aspect ratios $\lambda$ between 10 and 51. After rf evaporative cooling to the desired temperature, we wait for $1 \mathrm{sec}$ (with rf "shielding") to allow the system to reach an equilibrium state. We then switch off the trapping potential within $200 \mu$ s and allow a variable time of flight.

Figure 1 shows examples of experimental data for various temperatures $T<T_{c}$ and aspect ratios $\lambda$. The usual anisotropic expansion of the condensate related to the anisotropy of the confining potential is clearly visible in the absorption images. The line density profiles below reflect the parabolic shape of the BEC density distribution. Remarkably, we observe pronounced stripes in the density distribution in some cases shown in Fig. 1. On average these stripes are more pronounced at high aspect ratios of the trapping potential [Fig. 1(c)], high temperatures (bottom row of Fig. 1), and low atom numbers.

The appearance of stripes can be understood qualitatively as follows. As mentioned above, within the equilibrium state of a BEC in a magnetic trap the density distribution remains largely unaffected even if the phase fluctuates [8]. The reason is that the mean-field interparticle interaction prevents the transformation of local velocity fields provided by the phase fluctuations into modulations of the density. However, after switching off the trap, the mean-field interaction rapidly decreases and the axial velocity fields are then converted into the density (a)

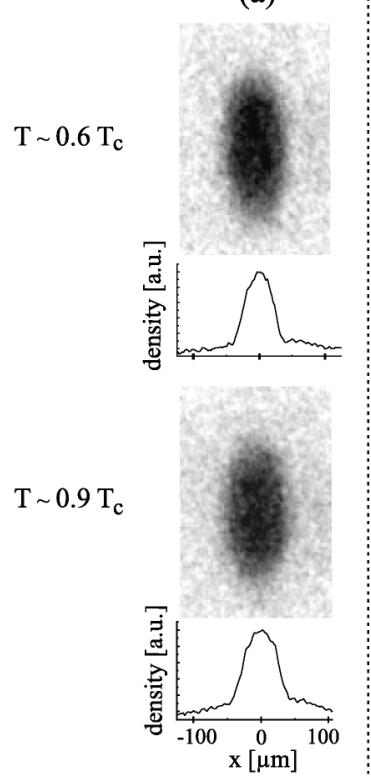

(b)

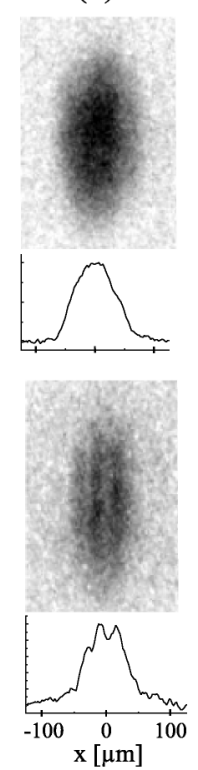

(c)
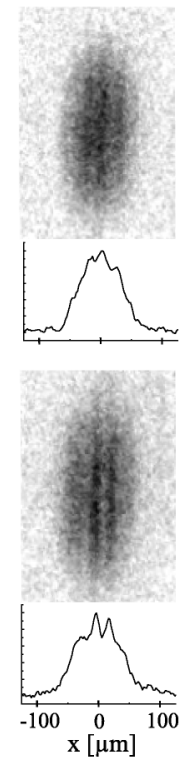

distribution. We have performed numerical simulations of the 3D Gross-Pitaevskii equation to understand quantitatively how phase fluctuations lead to the buildup of stripes in the density distribution. We assume that initially the condensate had an equilibrium density profile, and a random fluctuating phase $\phi(x)$. For elongated BECs [8] the phase can be represented as $\phi(x)=\sum_{j=1}^{\infty} \phi_{j}(x)$, where

$\phi_{j}(x)=\left[\frac{(j+2)(2 j+3) g}{4 \pi R^{2} L \epsilon_{j}(j+1)}\right]^{1 / 2} P_{j}^{(1,1)}\left(\frac{x}{L}\right) \frac{\left(\alpha_{j}+\alpha_{j}^{*}\right)}{2}$.

Here $\epsilon_{j}=\hbar \omega_{x} \sqrt{j(j+3) / 4}$ is the spectrum of lowenergy axial excitations [17], $P_{j}^{(1,1)}$ are Jacobi polynomials, $g=4 \pi \hbar^{2} a / m, a>0$ is the scattering length, and $R(L)$ is the radial (axial) condensate size. In Eq. (1), quasiparticle creation and annihilation operators have been replaced by complex amplitudes $\alpha_{j}$ and $\alpha_{j}^{*}$. To reproduce the quantum statistical properties of the phase, $\alpha_{j}$ and $\alpha_{j}^{*}$ were sampled as random variables with a zero mean value and $\left\langle\left|\alpha_{j}\right|^{2}\right\rangle=N_{j}$, where $N_{j}=\left[\exp \left(\epsilon_{j} / k_{B} T\right)-1\right]^{-1}$ is the occupation number for the quasiparticle mode $j$.

Alternatively, the formation of the stripes has been studied by using the local density approximation for the axial profile and by relying on the scaling approach [18] for the radial expansion of the cloud. The rescaled GrossPitaevskii equation was then linearized with respect to small fluctuations of the phase gradients and the density. Our method accounts for the transformation of initially phononlike excitations into particlelike ones and covers both the (initially) hydrodynamic and the (ultimately) freeparticle regimes of expansion. For a condensate which initially is in the Thomas-Fermi regime and has a chemical potential $\mu$, the radially integrated density fluctuations $\delta n(x)$ after a time of flight $t$ in the interval $\mu / \hbar \omega_{x}^{2} \gg$ $t \gg \mu / \hbar \omega_{\rho}^{2}$ are given by

$\frac{\delta n(x)}{n_{0}(x)}=2 \sum_{j} \sin \left[\frac{\epsilon_{j}^{2} t}{\hbar \mu\left[1-\left(\frac{x}{L}\right)^{2}\right]}\right]\left(\omega_{\rho} t\right)^{-\left(\epsilon_{j} / \hbar \omega_{\rho}\right)^{2}} \phi_{j}(x)$.

Here the profile $n_{0}(x)$ is the radially integrated density for the unperturbed condensate. From Eq. (2) one obtains a closed relation for the mean square density fluctuations $\left(\sigma_{\mathrm{BEC}} / n_{0}\right)^{2}$ by averaging $\left(\delta n / n_{0}\right)^{2}$ over different realizations of the initial phase. In the central part of the cloud $(x \approx 0)$ we find

$$
\left(\frac{\sigma_{\mathrm{BEC}}}{n_{0}}\right)^{2}=\frac{T}{\lambda T_{\phi}} \sqrt{\frac{\ln \tau}{\pi}}\left(\sqrt{1+\sqrt{1+\left(\frac{\hbar \omega_{\rho} \tau}{\mu \ln \tau}\right)^{2}}}-\sqrt{2}\right),
$$

where $\tau=\omega_{\rho} t$. In Eq. (3) we introduced a characteristic temperature $k_{B} T_{\phi}=15\left(\hbar \omega_{x}\right)^{2} N_{0} / 32 \mu$. For $T_{\phi}<T_{c}$ one expects the regime of quasicondensation for the initial cloud in the temperature interval $T_{\phi}<T<T_{c}$; i.e.,
FIG. 1. Absorption images and corresponding density profiles of BECs after $25 \mathrm{~ms}$ time of flight taken for various temperatures $T$ and aspect ratios $[\lambda=10(\mathrm{a}), 26(\mathrm{~b}), 51$ (c)].

$160406-2$ 
the regime where the coherence length is smaller than the condensate size [8]. The analytical expressions agree very well with the numerical simulations (see Fig. 2). Note that Eq. (3) provides a direct relation between the observed density fluctuations and the temperature, and thus can be used for thermometry at very low $T$.

To determine the amount of phase fluctuations experimentally, we systematically studied the formation and structure of stripes in the atomic density distribution as a function of the trapping potential and temperature. For each realization of a BEC, the observed density distribution was integrated along the radial direction and then fitted by a bimodal function with the integrated parabolic Thomas-Fermi distribution for the condensate fraction and a Gaussian for the thermal cloud. For each image we obtained standard deviations $\sigma_{\mathrm{BEC}}$ of the experimental data from the fit in the central region of the condensate fraction (half width of full size).

The temperature dependence of $\sigma_{\mathrm{BEC}}$ is shown in Fig. 3 for four different trap configurations with aspect ratios of $\lambda=10,26,36$, and 51. To account for shot-to-shot variations in the atom number, the standard deviations were normalized to the fitted peak density $n_{0}$ in the condensate. The dotted lines show the average detection noise $\sigma_{T} / n_{0}$ for data from each trap, obtained from the thermal wings of the cloud.

Since the initial phase of a Bose condensate is mapped into its density distribution after time of flight, the quantity $\sigma_{\mathrm{BEC}}$ is a direct measure of the initial phase fluctuations. Note, however, that this method reflects the instantaneous phase of the BEC at the time of release and, therefore, images taken at the same initial conditions can look sig-

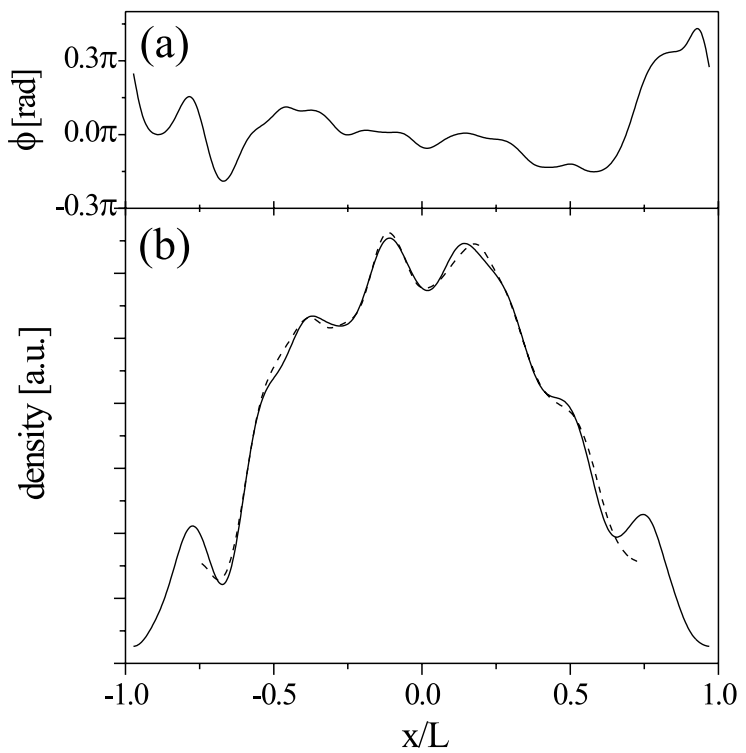

FIG. 2. (a) A typical initial phase distribution calculated for $\omega_{x}=2 \pi \times 14 \mathrm{~Hz}, \omega_{\rho}=2 \pi \times 508 \mathrm{~Hz}, N_{0}=2 \times 10^{5}$, and $T=0.5 T_{c}$. (b) Corresponding density profile after $25 \mathrm{~ms}$ time of flight from simulations (solid line) and analytical theory (dashed line). nificantly different. Indeed, we observe a large spread of our experimental data (see Fig. 3), which clearly demonstrates the statistical character of the phase fluctuations. Furthermore, our data characterize phase fluctuations for various trap geometries. For $\lambda=10$ the data fall into our detection noise and, therefore, the phase fluctuations are hardly observable. Considerably different images are observed for more elongated traps with $\lambda=26,36$, and 51. In these cases we observe significant deviations from the Thomas-Fermi distribution at high temperatures, which is a clear indication of the presence of phase fluctuations. For lower temperatures, reached by further evaporation, phase fluctuations become reduced due to both the reduced excitation spectrum at lower temperature and the increasing number of atoms in the condensate fraction. However, for even further evaporation the number of Bose-condensed atoms $N_{0}$ decreases leading to phase fluctuations in agreement with Eq. (3). In fact, it was very difficult to reduce the density modulation below the noise limit by rf evaporation in the case of our tightest trap with $\lambda=51$.

As observed in our experiment phase fluctuations for BECs in elongated traps continuously decrease with reducing $T$ and increasing $N_{0}$. It is not possible to determine a cutoff for the phase fluctuations; rather they decrease until they cannot be resolved below our noise limit. Hence all experiments with BECs at finite temperature in tightly confining elongated potentials will be subject to axial phase fluctuations.

In order to obtain general information about the phase fluctuations, we average the observed standard deviations over many realizations within a small temperature and particle number interval. This allows us to use Eq. (3) and to compare directly the averaged measured value of

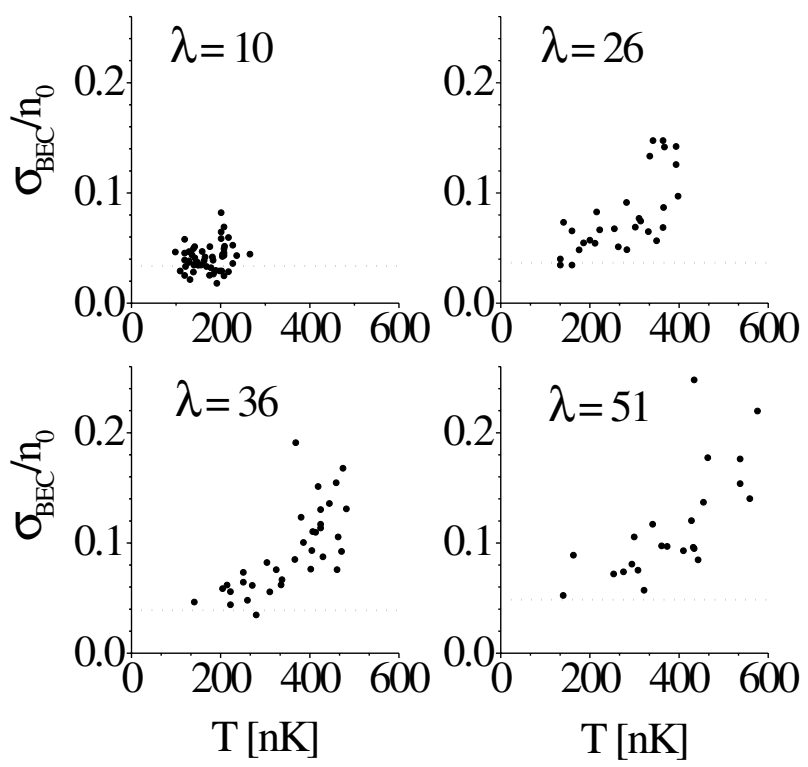

FIG. 3. Measurement of $\sigma_{\mathrm{BEC}} / n_{0}$ versus temperature in four different trap geometries. The dotted lines represent the average detection noise $\sigma_{T} / n_{0}$. All data were taken for $T<T_{c}$. 


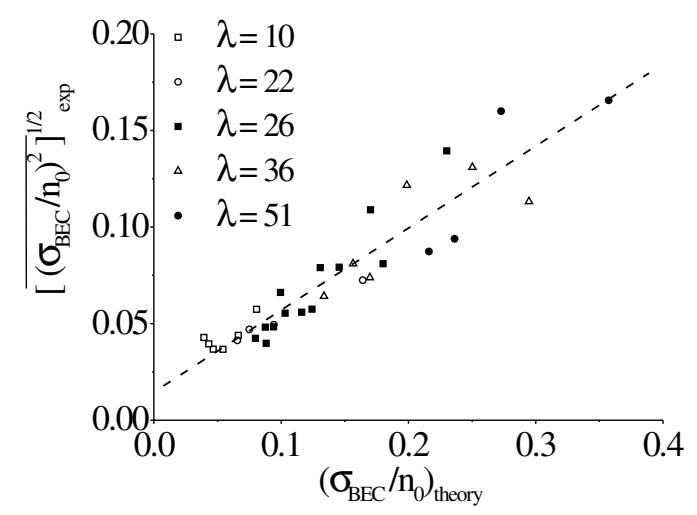

FIG. 4. Average standard deviation of the measured line densities $\left[\overline{\left(\sigma_{\mathrm{BEC}} / n_{0}\right)^{2}}\right]_{\exp }^{1 / 2}$ compared to the theoretical value of $\left(\sigma_{\mathrm{BEC}} / n_{0}\right)_{\text {theory }}$ obtained from Eq. (3). The dashed line is a fit to the experimental data.

$\left[\overline{\left(\sigma_{\mathrm{BEC}} / n_{0}\right)^{2}}\right]_{\exp }^{1 / 2}$ to the predicted value of $\left(\sigma_{\mathrm{BEC}} / n_{0}\right)_{\text {theory }}$ for various values of $T, N_{0}, \omega_{x}, \omega_{\rho}$, and $t$ (see Fig. 4). The theoretical value takes also the limited experimental imaging resolution into account.

Our experimental results follow the expected general dependence very well. With the direct link of the phase fluctuations in the magnetic trap to the observed density modulation given by Eq. (3), our data therefore confirm the predicted general behavior of phase fluctuations in elongated BECs. However, the measured values are by a factor of approximately 2 smaller than those predicted by theory. This discrepancy could be due to, e.g., a reduction of the observed contrast caused by a small tilt in the detection laser beam with respect to the radial stripes [19]. Most of the observed experimental data, which exhibit fluctuations well above the noise limit, correspond to $T>T_{\phi}$. This implies that the measurements were performed in the regime of quasicondensation; i.e., the phase coherence length $l_{\phi}=L T_{\phi} / T$ of the initial condensate was smaller than the axial size $L$. For instance, for $\lambda=51, T=0.5 T_{c}$, and $N_{0}=3 \times 10^{4}$, one obtains $l_{\phi} \approx L / 3$.

In conclusion, we have presented detailed experimental and theoretical studies of a BEC state with fluctuating phase. The flexible method of ballistic expansion allows one to measure phase fluctuations under various experimental conditions, especially for various trap geometries. Bragg spectroscopy can also be used to measure the influence of phase fluctuations on the momentum distribution of particles in the axial direction, but this method is difficult to apply to very elongated traps. In addition, the ballistic expansion for very long times can visualize extremely small phase fluctuations not accessible to the usual resolution of Bragg spectroscopy. By measuring the phase fluctuations and comparing the temperature with $T_{\phi}$ we have demonstrated instances where the phase coherence length was smaller than the axial size of the condensate; i.e., the initial cloud was in the quasicondensate state. Our results set severe limitations on applications of BECs in interferometric measurements, and for guided atom laser beams. Our experimental method combined with the theoretical analysis [Eq. (3)] provides a method of BEC thermometry. Further studies of quasicondensates, e.g., with respect to superfluid properties, will be necessary to obtain a full understanding of the phase coherence properties of ultracold atomic gases.

This work is supported by SFB407 of the Deutsche Forschungsgemeinschaft. D. S. P. and G. V.S. acknowledge support from the Alexander von Humboldt Foundation, from the Dutch Foundations NWO and FOM, and from the Russian Foundation for Basic Research.

[1] M. J. Anderson et al., Science 269, 198 (1995); K. B. Davis et al., Phys. Rev. Lett. 75, 3969 (1995); C. C. Bradley et al., Phys. Rev. Lett. 78, 985 (1997); D. Fried et al., Phys. Rev. Lett. 81, 3811 (1998).

[2] J. Stenger et al., Phys. Rev. Lett. 82, 4569 (1999).

[3] E. W. Hagley et al., Phys. Rev. Lett. 83, 3112 (1999).

[4] D. S. Petrov, G. V. Shlyapnikov, and J.T. M. Walraven, Phys. Rev. Lett. 85, 3745 (2000).

[5] D. S. Petrov, M. Holzmann, and G. V. Shlyapnikov, Phys. Rev. Lett. 84, 2551 (2000).

[6] Yu. Kagan et al., Phys. Rev. A 61, 43608 (2000).

[7] A. I. Safonov et al., Phys. Rev. Lett. 81, 4545 (1998).

[8] D. S. Petrov, G. V. Shlyapnikov, and J.T.M. Walraven, Phys. Rev. Lett. 87, 050404 (2001).

[9] Here we refer to the equilibrium state of the system, which has a fluctuating phase, in contrast to the notion of a "quasicondensate" appearing as an intermediate state during the formation process of a BEC [10,11].

[10] Yu. Kagan, in Bose-Einstein Condensation, edited by A. Griffin, D. Snoke, and S. Stringari (Cambridge University Press, Cambridge, 1995), pp. 202-225, and references therein.

[11] W. Ketterle and H.-J. Miesner, Phys. Rev. A 56, 3291 (1997), and references therein.

[12] J. Denschlag et al., Phys. Rev. Lett. 82, 2014 (1999); J. Reichel et al., Phys. Rev. Lett. 83, 3398 (1999); D. Müller et al., Phys. Rev. Lett. 83, 5194 (1999).

[13] I. Bloch, T. W. Hänsch, and T. Esslinger, Nature (London) 403, 166 (2000).

[14] K. Bongs et al., Phys. Rev. Lett. 83, 3577 (1999).

[15] S. Burger et al., Phys. Rev. Lett. 83, 5198 (1999).

[16] K. Bongs et al., Phys. Rev. A 63, R31602 (2000).

[17] S. Stringari, Phys. Rev. A 58, 2385 (1998).

[18] Yu. Kagan, E. L. Surkov, and G. V. Shlyapnikov, Phys. Rev. A 54, R1753 (1996); Y. Castin and R. Dum, Phys. Rev. Lett. 77, 5315 (1996).

[19] We have calculated that for our parameters a tilt of approximately $4^{\circ}$ is already sufficient to reduce the observed contrast by a factor of 2 . 